\title{
Politique
}

\section{La recherche en administration publique au Québec : 1960-1980}

\section{Carolle Simard}

Numéro 11, hiver 1987

L’État privé

URI : https://id.erudit.org/iderudit/040547ar

DOI : https://doi.org/10.7202/040547ar

Aller au sommaire du numéro

Éditeur(s)

Société québécoise de science politique

ISSN

0711-608X (imprimé)

1918-6584 (numérique)

Découvrir la revue

Citer cet article

Simard, C. (1987). La recherche en administration publique au Québec :

1960-1980. Politique, (11), 73-106. https://doi.org/10.7202/040547ar d'utilisation que vous pouvez consulter en ligne.

https://apropos.erudit.org/fr/usagers/politique-dutilisation/ 


\title{
LA RECHERCHE EN ADMINISTRATION PUBLIQUE AU QUÉBEC: 1960-1980*
}

\author{
Carolle Simard \\ Université du Québec à Montréal
}

L'objectif de cet article est de faire la revue des travaux faits au Québec, en administration publique, à travers leur recension. Plus précisément, il s'agit d'effectuer un inventaire de la recherche réalisée à l'extérieur de l'administration québécoise au cours de cette période: 1960-1980. Cet inventaire ne concerne que les travaux complétés dans le cadre universitaire francophone et où les politologues, mais aussi les juristes, les sociologues, les économistes et les psychologues ont contribué à développer un système de recherche qui s'intéresse aux divrs aspects de la vie administrative: sociologie des fonctionnaires, fonctionnement des institutions, processus décisionnel, problèmes liés au contrôle, etc.

Deux questions principales seront successivement abordées. La première concerne la recherche elle-même dans ses caractéristiques essentielles, par le biais de l'étude de ses tendances et de son évolution. C'est à une reconstitution des éléments du corpus de connaissance via ces interrogations: - qu'est-ce qu'on étudie et

* Cette recherche a reçu l'appui financier du Fonds institutionnel de recherche de I'UQAM. L'auteur remercie Jacques Léveillée pour ses critiques et commentaires sur une première version de ce texte. 
comment? - que nous procèderons d'abord. La seconde question porte sur les enjeux du système de recherche, dès lors que ces enjeux constituent un guide pour le repérage des changements administratifs affectant l'État. En clair, il s'agit de voir s'il existe une relation entre l'évolution du corpus de connaissance et les changements administratifs qui ont pris lieu et place au sein de l'appareil d'État québécois.

\section{Méthodologie de la recherche}

En entreprenant ce bilan nous n'avions pas de définition très précise de la recherche relative à l'administration publique québécoise. Tous les travaux dont le titre suggérait l'étude d'un problème lié à l'organisation et au développement de l'administration publique québécoise aux niveaux scolaire, municipal et provincial ont d'abord été retenus. Puisque nous posions que la recherche sur l'administration publique n'était pas rattachée à une discipline unique ces secteurs ont été inventoriés: science politique, sociologie, relations industrielles, économie, science de la gestion, droit. Tant à Québec qu'à Montréal, les centres de recherche et de documentation universitaires ont été visités et les fichers des bibliothèques parcourus (voir la liste tableau 1). En outre, nous avons dépouillé ces revues:

Revue canadienne de science politique

Revue d'administration publique du Canada

Revue administration et Santé

Revue administration hospitalière et sociale

Revue maintenant

Revue internationale des sciences administratives

Revue relations industrielles

Cahiers de droit

Annuaire du Québec

Revue du barreau 
Revue internationale de droit comparé

L'Actualité économique

Revue commerce

Revue de la société d'études et d'expansion

Pour Québec et Montréal, plus de 1000 recherches ont été rencensées entre 1960 et 1980 . À la fin de cette première phase certains constats ressortaient: si trois recherches sur quatre avaient été effectuées entre 1970 et 1980 c'était surtout à Québec, du moins à partir de 1975, que la plupart des recherches étaient initiées et menées. Avant 1970, c’est Montréal qui monopolisait ce secteur de recherche.

L'analyse des travaux de recherche a suivi la phase d'inventaire. Environ une recherche sur trois fut analysée. Nous avons retenu le pourcentage de $30 \%$, tant pour les périodes d'étude que pour les centres de recherche (voir tableau 1). Bien qu'il fut parfois

TABLEAU 1

Périodes de recherches

\begin{tabular}{l|c|c|c|c|c}
\hline $\begin{array}{l}\text { CENTRES DE } \\
\text { RECHERCHES }\end{array}$ & $1960-1965$ & $1966-1970$ & $1971-1975$ & $1976-1980$ & TOTAL \\
\hline UNIVERSITÉ DE & & & & & \\
MONTRÉAL & & & & & \\
A) Centres de recherches & & & & & \\
- Adm. scol. & 2 & 3 & 2 & 1 & 6 \\
- CRDP & 2 & & 6 & 6 & 14 \\
- CDDE & & & 2 & & 4 \\
- CREA & 2 & 4 & 2 & 8 & 2 \\
ERIS & 5 & 2 & 4 & 2 & 13 \\
- SRDSP & 2 & & 2 & 1 & 14 \\
B) H.E.C. & 13 & 18 & 25 & 18 & 74 \\
C) Thèses & & & 5 \\
\hline TOTAL & & & & & 5 \\
\hline
\end{tabular}




\begin{tabular}{|c|c|c|c|c|c|}
\hline $\begin{array}{l}\text { UNIVERSITÉ LAVAL } \\
\text { A) Centres de recherches } \\
\text { - CRAD } \\
\text { - LEPA } \\
\text { - LJA } \\
\text { - LR APS } \\
\text { B) Dép. rel. indust. } \\
\text { C) Thèses }\end{array}$ & & $\begin{array}{l}6 \\
3 \\
1 \\
2 \\
9\end{array}$ & $\begin{array}{l}5 \\
6 \\
1 \\
3 \\
8\end{array}$ & $\begin{array}{r}1 \\
8 \\
5 \\
2 \\
2 \\
26\end{array}$ & $\begin{array}{r}1 \\
19 \\
14 \\
4 \\
7 \\
43\end{array}$ \\
\hline TOTAL & & 21 & 23 & 44 & 88 \\
\hline TOTAL CUMULATIF & 13 & 39 & 48 & 62 & 162 \\
\hline $\begin{array}{l}\text { UNIVERSITÉ DU QUÉBEC } \\
\text { - INRS-URB } \\
\text { - UQAM } \\
\text { - CDEA } \\
\text { - LABREV } \\
\text { - Dép. sc. pol. } \\
\text { - ENAP (Mtl.) } \\
\text { - ENAP (Québec) }\end{array}$ & & & $\begin{array}{l}4 \\
2 \\
4 \\
8 \\
9\end{array}$ & $\begin{array}{r}2 \\
6 \\
15 \\
15\end{array}$ & $\begin{array}{c}9 \\
2 \\
2 \\
10 \\
23 \\
24\end{array}$ \\
\hline TOTAL & - & - & 27 & 43 & 70 \\
\hline TOTAL CUMULATIF & 13 & 39 & 75 & 105 & 232 \\
\hline REVUES ET VOLUMES & 8 & 9 & 28 & 3 & 48 \\
\hline $\begin{array}{l}\text { GRAND TOTAL } \\
\text { CUMULATIF }\end{array}$ & 21 & 48 & 103 & 108 & 280 \\
\hline
\end{tabular}

difficile de respecter ce pourcentage sur une base quinquennale, près de 300 recherches ont pu être analysées. L'écart est cependant relatif puisqu'il est de plus ou moins cinq pour cent et ce, dans tous les cas.

La sélection des travaux une fois terminée, se posait le problème de savoir quelle était la meilleure façon de rendre cohérent ce qui, avouons-le, apparaissait relativement diversifié, tant au niveau 
de la forme que du contenu. Mais, il nous fallait auparavant répondre à une autre question à savoir: qu'est-ce que l'étude de l'administration publique? S'agit-il d'une discipline reposant sur une théorie dont découle un point de vue propre sur les phénomènes administratifs? S'agit-il plus simplement de l'étude de l'application de la politique davantage que de sa formulation?

Tandis que l'amorce d'un débat épistémologique risquait fort de paraître un peu gratuit pour la suite de notre propos, d'autant que d'autres ont déà longuement discouru sur la question, sans jamais parvenir à esquiver complètement le problème de la discipline de rattachement relative à l'étude des problèmes administratifs ${ }^{1}$, il fallait nous débarrasser de cette vision duale qui ressort trop souvent des principaux travaux d'administration publique. En effet, d'un côté nous avons des études marquées par «l'ingénieurisme" administratif ${ }^{2}$ dès lors que l'administration «n'est qu'une gigantesque machine dont il faut perfectionner les rouages pour en faciliter le rendement et en améliorer la cohérence». De l'autre, nous avons des travaux qui ne considèrent que la question du pouvoir, "comment il est exercé, par qui et dans quel but", cet aspect constituant le fond du problème ${ }^{3}$. Ainsi, à la vision instrumentale de l'administration s'oppose celle qui lui refuse une spécificité, dès lors que toute l'administration est politique. Bien sûr, entre ces deux extrêmes coexistent plusieurs positions intermédiaires, tandis qu'il n'est guère facile de lever un peu le voile sur les théories dont elles relèvent.

Certes, donner de l'administration publique une définition a priori eût été plus simple d'autant que n'importe quel manuel

1. Notamment Jacques Chevallier et Danièle Loschak, Traité de science administrative, (2 tomes), Paris, Librairie générale de droit et de jurisprudence, 1978.

2. Bruno Jobert, "L'État en action: l'apport des politiques publiques», article à paraître dans la Revue française de science politique, 1985.

3. Peta Sheriff, tel que cité par Kenneth Kernaghan, «L'administration publique canadienne: situation actuelle et perspectives d'avenir ", Administration publique du Canada, vol 25, no 4, hiver 1982, p. 463. 
d'administration publique en propose une. De surcroît, il existe une classification de la bibliographie de l'Institut d'administration publique du Canada, laquelle aurait pu être exploitée avec avantage sans doute ${ }^{4}$. Enfin, à l'occasion du $25^{\text {ème }}$ anniversaire d'Administration publique du Canada, la revue de l'Institut d'administration publique du Canada, Kenneth Kernaghan fait une analyse du contenu de la revue depuis sa fondation ${ }^{5}$. Les articles sont répertoriés par sujet et par auteur, d'après une "classification des sujets qui se base essentiellement sur le contenu habituel des manuels d'administration publique» ${ }^{6}$. Quant aux auteurs, deux grandes catégories les distinguent: les universitaires, les praticiens et autres.

En France, l'Institut français des sciences administratives a, lors de journées d'études, procédé à diverses tentatives de regroupement. C'est dans ce cadre que, François Gazier, a identfié quatre grands secteurs de la recherche administrative: la recherche fondamentale, la recherche au service de l'enseignement, la recherche au service de la coopération, les perspectives de la recherche au service des administrations ${ }^{7}$. Quelques années auparavant, Bernard Gournay avait classé les enquêtes et recherches en fonction du niveau auquel elles se situent. Parlant des centres de recherche spécialisés, essentiellement le Centre de recherche sur l'administration économique et l'aménagement du territoire de Grenoble et le Centre de recherches administratives de la Fondation nationale des sciences politiques, il attribue trois thèmes d'attache au Centre de Grenoble: centralisation et décentralisation dans les sociétés industrielles, les institutions administratives chargées de l'amé-

4. G. Julien et W. E. Grasham, «Bibliographie», Administration publique du Canada, Supplément 2, 1973-1975.

5. «L'administration publique canadienne: situation et perspectives d'avenir ", op. cit.

6. Ibid., p. 459.

7. François Gazier, "Perspectives de la recherche administrative", Les problèmes actuels de la recherche administrative, Paris, Éditions Cujas, Cahier no 6, 1971, pp. 31-43. 
nagement du territoire et de la planification régionale, l'administration économique et les dirigeants du secteur public. Quant au Centre de Paris il se spécialise, toujours selon Gournay, dans l'étude de ces secteurs: l'administration locale, les corps et catégories de fonctionnaires, l'action administrative ${ }^{8}$. Précisons toutefois qu'il existe aujourd'hui deux autres centres de recherche spécialisés, soit le Centre d'étude et de recherche sur la vie locale et le Centre universitaire de recherches administratives et politiques de Picardie.

Mais, procéder au regroupement et au croisement des thèmes et des institutions ne permet guère de dépasser la conception organique de la recherche administrative. Une telle conception n'est-elle pas devenue obsolète eu égard au fait, maintes fois démontré maintenant par les études sociologiques ${ }^{9}$, que la réalité administrative ne saurait être confondue avec la rationalité des textes. "L'administration est à l'image de Janus: elle a deux images, l'un est bureaucratique, l'autre est politique. La logique politique marque fortement son action ${ }^{10}$.

La difficulté de marquer un point de rupture entre le politique et l'administratif devait être contournée. De surcroît, il fallait rendre compte de la diversité des champs d'intervention des administrations publiques modernes. L'État traditionnel a des missions politiques et de souveraineté. Pour sa part, l'État moderne, le «tout» État, agit également aux plans économique, social et culturel. La typologie de Bernard Gournay ${ }^{11}$, basée sur des champs sectoriels de politique - il attribue quatre missions principales à l'administration: les missions de souveraineté, les missions éco-

8. Bernard Gournay, «La recherche administrative dans les instituts d'études politiques ", La recherche administrative en France, Paris, Éditions Cujas, Cahier no 3, 1968, pp. 21-39.

9. Sur cette question, voir le dernier ouvrage de François Dupuy et Jean-Claude Thoenig, L'administration en miettes, Paris, Fayard, 1985.

10. Ibid., p. 178.

11. Bernard Gournay, Introduction à la science administrative, Paris, Armand Colin, 1966, pp. 18-19. 
nomiques, les missions sociales, les missions éducatives et culturelles - nous est apparue relativement appropriée, compte tenu des objectifs fixés.

Bien sûr, dans l'absolu, toute grille de lecture peut en remplacer une autre ${ }^{12}$ mais, parce que l'administration n'est ni un instrument de l'État, ni en situation d'extériorité totale par rapport au politique, cette classification demeurait la plus opérante.

Ne restait plus, désormais, que la question des problématiques les plus courantes retenues par les chercheurs, et dont l'objectif visait à identifier les axes principaux de la recherche administrative. D'emblée, nous avons éliminé une analyse reposant sur les théories du pouvoir parce que celles-ci se situent toujours en arrière plan. En outre, une telle façon de procéder soulevait d'énormes difficultés dès lors que les divisions retenues risquaient d'être tantôt acceptées, tantôt rejetées. Cirtes, partir des problématiques signifie nécessairement procéder à des distinctions. Toute distinction impliquant une construction, il se trouve qu'elles ne sont ni évidentes, ni même acceptées par tous et toutes. Nous devions prendre en compte les problématiques les plus courantes sans que celles-ci renvoient nécessairement à une conception homogène. Et, comme la plupart des manuels s'accordent plus ou moins sur trois ou quatre approches, il nous a semblé pertinent de retenir celles faisant l'objet d'un plus large consensus ${ }^{13}$. Il s'agit de l'approche

12. Dans une première version de ce texte, on a critiqué le fait que j'utilisais la grille de Gournay, me reprochant de l'avoir préférée à la classification de la bibliographie de l'Institut d'administration publique du Canada.

13. Travailler à distinguer les problématiques est plus difficile qu'on le croit. L'Institut français des sciences administratives, lors des journées d'étude consacrées à la recherche administrative en France n'a guère abordé cette question, le problème institutionnel demeurant au centre des débats. Pour sa part, Kenneth Kernaghan dans «L'administration publique canadienne: situation actuelle et perspectives d'avenir", y consacre quatre lignes. Il écrit: "D'une manière générale, la majorité des auteurs $(75,8 \%)$ ont traité le thème de leur article selon une méthode historico-descriptive; $20,8 \%$ des articles ont reflété un ton normatif/descriptif et $3,4 \%$ seulement des auteurs ont opté pour un traitement behavioriste/empirique», op. cit., p. 460 . 
juridico-politique, l'approche économique, l'approche managériale et l'approche sociologique ${ }^{14}$. Chacune appréhende le processus administratif différemment et renvoie à une vision hétérogène de l'administration. Cette dernière est tantôt analysée comme un fait social, tantôt comme une entitée subordonnée.

\section{Le corpus de connaissance}

En reprenant le tableau 1, on peut faire certaines remarques quantitatives. D'abord, qu'il y a eu progression du nombre de travaux de 1960 à 1980. Cette progression est relativement importante puisqu'au cours des années soixante-dix, trois fois plus de travaux ont été recensés que lors de la période précédente. La présence accrue de l'Université Laval de même que la création de l'Université du Québec semblent responsables de cet accroissement. De plus, la mise sur pied, au cours des années soixante-dix, de centres de recherche spécialisés a sans doute exercé un effet d'entraînement. Notre méthode de collecte de données ne nous permet pas d'établir avec précision le nombre d'études complétées au sein de chaque discipline. Cependant, la plupart des travaux analysés proviennent d'abord des politologues et des juristes, puis des sociologues ainsi que des spécialistes des sciences de la gestion.

Les champs étudiés

Muni de la grille de Bernard Gournay sur les missions de l'administration voyons donc quels sont les champs constitués ou encore en formation. Encore une fois, nous sommes conscient qu'une telle façon de procéder est relativement arbitraire au sens où certaines recherches auraient pu être classées dans plus d'une mission. Mais, afin de ne pas compliquer l'analyse inutilement

14. Jacques Chevallier et Danièle Loschak, Traité de science administrative, tome 1 , op. cit., font le point sur cette question. 
et lorsque cette difficulté s'est présentée nous avons classé les recherches en cause dans la rubrique qui se rapprochait le plus de la fonction dominante exercée par l'institution étudiée. Une première classification des recherches analysées donne ce résultat: 120 recherches concernent les missions de souveraineté, 79 les missions économiques, 46 les missions sociales, 33 les missions éducatives et culturelles. Certains traits ressortent de ce classement et, à travers l'analyse fouillée de chaque mission, se profilent ces caractéristiques.

Les missions de souveraineté

Aux trois sous-divisions de Gournay nous avons ajouté celle des missions administratives et financières. Le nombre de recherches autour de ces questions étant relativement important il nous semblait pertinent d'en cerner les particularités. La grille d'analyse relative aux missions de souveraineté se lit ainsi:

$1^{\circ} \quad$ Les missions de souveraineté externe:

- la défense (ou sécurité) nationale;

- la conduite des relations extérieures.

$2^{\circ} \quad$ Les missions de souveraineté interne:

- la police (appelée aussi sécurité intérieure);

- la justice (y compris l'état-civil).

$3^{\circ}$ Les missions proprement politiques:

- le fonctionnement des institutions politiques (élections, assemblées);

- les relations avec les institutions religieuses;

- l'information de l'opinion à des fins politiques.

$4^{\circ}$ Les missions administratives et financières:

- le fonctionnement des institutions;

- les modes de gestion ${ }^{15}$.

15. Les sous-divisions 1-2-3 sont celles de Gournay, ibid. 
Si les recherches traitant des missions de souveraineté sont nombreuses, elles sont néanmoins fort inégalement distribuées à l'intérieur des quatre sous-divisions. Sauf exception, la plupart des travaux sont regroupés soit au sein des missions de souveraineté interne, soit au sein des missions administratives et financières.

Sous la rubrique missions de souveraineté interne nous retrouvons treize recherches; toutes relevant du domaine de la justice. La thématique qui domine est celle du protecteur du citoyen (cinq recherches). Les autres thèmes concernent l'administration générale de la justice et le droit administratif. Presque toutes les recherches ont été réalisées entre 1970 et 1980, soit cinq entre 1976-1980 et quatre entre 1971-1975. Pour l'essentiel, il s'agit d'articles rédigés par des professeurs de droit qui se limitent le plus souvent à décrire tantôt la législation, tantôt les institutions.

Quant aux missions administratives et financières elles regroupent 101 recherches. La quasi-totalité d'entre elles traitent du fonctionnement des institutions aux niveaux central, régional ou encore municipal. Cinquante recherches, dont trente-huit réalisées entre 1970 et 1980, étudient ce qui se passe au centre: l'organisation générale du gouvernement et son fonctionnement, les questions liées aux personnels publics, les éléments de contrôle institués ou à mettre en place. Trois organismes sont l'objet d'un plus grand nombre de travaux, soit le Conseil du trésor, le ministère de la Fonction publique et la Commission de la fonction publique. L'analyse des cinquante recherches montre qu'il s'agit majoritairement d'articles que complètent quelques ouvrages généraux sur le fonctionnement et l'organisation de l'administration gouvernementale.

Près d'une recherche sur deux a été menée tantôt par des professeurs de l'Université Laval, tantôt par des chercheurs de l'ENAP. Pour leur part, les professeurs de l'Université de Montréal ont produit une dizaine de recherches. 
Les recherches sur la périphérie portent soit sur les régions (douze travaux), soit sur les municipalités (vingt-cinq travaux). La recherche municipale a été particulièrement féconde entre 1965 et 1975, période au cours de laquelle une quinzaine d'études ont pu être recensées. À part l'administration municipale de Montréal, laquelle fait l'objet de quelques travaux, c'est le problème plus général de l'évolution des structures municipales découlant du partage des pouvoirs entre la province et les municipalités qui est le plus souvent décrit et étudié. Après 1975, on note que les chercheurs commencent à s'intéresser au problème de la fiscalité municipale, notamment à la gestion et au contrôle.

En ce qui concerne les travaux sur la région (une douzaine), ils traitent autant des régions administratives en général que de certaines régions en particulier. Dans ce dernier cas, ce sont l'Outaouais et le Nouveau-Québec qui ont été plus souvent retenus par les chercheurs, préoccupés par l'étude des processus de décentralisation et de déconcentration dans le cadre de la politique de régionalisaion du gouvernement Lévesque.

Les recherches et travaux relatifs aux modes de gestion sont numériquement peu importants; on en compte quatorze. Les chercheurs s'intéressent notamment au problème de la rationalisation des choix budgétaires, à son contrôle et à son implantation. On y traite également de la gestion ministérielle de même que des dépenses gouvernementales. L'organisme le plus souvent étudié est le Conseil du trésor. Sauf une, toutes ces études ont été initiées entre 1970 et 1980. L'ENAP a complété une recherche sur deux.

Finalement, cinq recherches peuvent être classées sous la rubrique missions proprement politiques. Presque toutes ont été réalisées entre 1970 et 1980 tandis que le thème dominant est celui des cabinets $^{16}$.

16. Il y a aussi une recherche traitant de ce que Gournay appelle «l'information de l'opinion à des fins politiques". 
Le tableau 2 donne un aperçu des champs que les chercheurs ont privilégiés. Quelques thèmes ressortent, ainsi que certaines institutions.

Les missions économiques

La grille de Gournay établit cette division au niveau des missions économiques:

$1^{\circ} \quad$ Les attributions de l'État relatives à la monnaie

$2^{\circ} \quad$ Les actions spécifiques:

- dans les différents secteurs de la vie économique (énergie, mines, transports, télécommunications, industries de transformation, agriculture et pêche, services);

- à propos des diverses fonctions ou problèmes communs à tous les secteurs (recherche appliquée et productivité, main-d'œuvre et formation professionnelle, investissement, crédit, prix, fiscalité, commerce extérieur, etc.).

$3^{\circ}$ La coordination générale de la politique économique et financière (en y incluant la coordination dans l'espace: aménagement du territoire).

Soixante-dix-neuf recherches ont été classées dans cette catégorie, soit quarante-huit dans la section «actions spécifiques» et trente et une au niveau de la "coordination générale». Il est sans doute normal qu'il n'y ait aucune recherche dans le domaine de la monnaie, puisque cette prérogative est du ressort exclusif du gouvernement fédéral. Environ $60 \%$ des recherches inventoriées étudient les actions spécifiques de l'État québécois dans le domaine économique, cependant que deux recherches sur trois s'intéressent aux secteurs de la vie économique. Exception faite de trois recherches, l'ensemble des travaux porte sur quatre secteurs principaux: les ressources naturelles (énergie, mines, terres et forêts), l'agriculture, les transports, les télécommunications. 
TABLEAU 2

Missions de souveraineté

\begin{tabular}{|c|c|c|c|c|}
\hline & NOMBRE & CHAMPS ÉTUDIÉS & THÈMES & $\begin{array}{c}\text { INSTITUTIONS } \\
\text { DES CHERCHEURS }\end{array}$ \\
\hline $\begin{array}{l}\text { Missions de souveraineté } \\
\text { externe }\end{array}$ & 13 & $\begin{array}{l}\text { - Administration générale de } \\
\text { la justice } \\
\text { - Droit administratif }\end{array}$ & $\begin{array}{l}\text { - Protecteur du citoyen } \\
\text { — La législation }\end{array}$ & $\begin{array}{l}\text { Université de } \\
\text { Montréal }\end{array}$ \\
\hline $\begin{array}{l}\text { Missions proprement } \\
\text { politiques }\end{array}$ & 5 & & — Les cabinets ministériels & \\
\hline $\begin{array}{l}\text { Missions administratives et } \\
\text { financières }\end{array}$ & $\begin{array}{l}50 \\
37\end{array}$ & $\begin{array}{l}\text { - Organisation générale du } \\
\text { gouvernement } \\
\text { - Évolution des structures } \\
\text { municipales } \\
\text { - Rapports centre-périphérie } \\
\text { - La politique de } \\
\text { régionalisation } \\
\text { - Les modes de gestion }\end{array}$ & $\begin{array}{l}\text { - Les personnels publics } \\
\text { - Le contrôle } \\
\text { — L'administration } \\
\text { municipale de } \\
\text { Montréal } \\
\text { — La fiscalité municipale } \\
\text { _ La gestion } \\
\text { - Le contrôle } \\
\text { — La rationalisation des } \\
\text { choix budgétaires }\end{array}$ & $\begin{array}{l}\text { Université Laval, } \\
\text { ENAP, Université de } \\
\text { Montréal } \\
\text { Université de } \\
\text { Montréal, } \\
\text { Université Laval }\end{array}$ \\
\hline
\end{tabular}


1) Les actions spécifiques dans la vie économique

a) Les ressources naturelles

Six recherches concernent l'Hydro-Québec et l'énergie hydroélectrique; six autres analysent dives aspects de la gestion des forêts publiques; une traite de la faune québécoise, une autre du secteur minier. Onze recherches sur quatorze ont été réalisées entre 1971 et 1980, tant par des chercheurs de l'Université Laval (thèses de maîtrise et recherches de professeurs) que de l'ENAP.

\section{b) Agriculture}

Seulement quatre recherches pour ce secteur d'activités, dont trois ont été complétées entre 1970 et 1980.

\section{c) Transports}

Sept recherches portent sur cette question. Le thème dominant est celui du transport en commun dans les zones métropolitaines. Cinq recherches ont été menées entre 1976 et 1980.

\section{d) Télécommunications}

Sur cinq recherches analysées, quatre ont été initiées après 1970. Elles concernent Radio-Québec ou encore les infrastructures de télécommunication québécoise.

Les différents secteurs de la vie économique ne semblent pas présenter le même intérêt pour les chercheurs. En effet, on constate que certains pans de la vie économique sont relativement délaissés, notamment les services, de même que les industries de transformation. Mais, quel que soit le secteur retenu, on note que la recherche s'est surtout développée à partir de 1970 . 


\section{2) Les autres actions spécifiques}

Pour leur part, les recherches traitant des diverses fonctions ou problèmes communs à tous les secteurs portent essentiellement sur le domaine du travail. Sont analysées les question liées à la négociation collective, aux règlements des griefs et à la loi du salaire minimum. Sur quatorze recherches, une seulement a été complétée entre 1976 et 1980. Deux institutions ont été particulièrement actives dans ce secteur de recherche: le département des relations industrielles de l'Université Laval et l'École des hautes études commerciales de Montréal. Les organismes les plus souvent cités sont la Commission du salaire minimum, le Tribunal du travail et la Commission des relations de travail. Dans leur très grande majorité, ces travaux se contentent de décrire et de commenter, parfois de critiquer, les problèmes issus de l'application des lois relatives au travail et à la main-d'œuvre ainsi que ceux liés au partage de juridiction entre les organismes concerns.

\section{3) La coordination générale de la politique économique et financière}

La recherche sur la coordination générale de la politique économique et financière couvre divers aspects de cette question. Les trente et une recherches répertoriées ont été classées selon trois rubriques: aménagement du territoire, politique économique et politique financière.

\section{a) Aménagement du territoire}

Sur dix recherches, huit ont été initiées après 1970. Les organismes les plus souvent mentionnés sont l'OPDQ et la Communauté urbaine de Québec. Une recherche sur deux est une thèse de maîtrise, complétée à l'Université Laval. 


\section{b) Politique financière}

Les thèmes qu'étudient ces onze recherches sont extrêmement variés, cependant que les travaux portant sur la dette publique sont relativement plus importants. La plupart des recherches ont été faites entre 1971 et 1975; l'ENAP et l'École des hautes études commerciales de Montréal sont responsables de près de la moitié de ces études.

\section{c) Politique économique}

Il n'y a pas de thématique générale pour les neuf recherches classées sous cette rubrique. À l'exception de deux recherches, toutes ont été complétées entre 1971 et 1975, tandis que les institutions d'appartenance des chercheurs sont quasiment aussi nombreuses que les sujets analysés.

Somme toute, les recherches relatives au champ économique de l'État sont assez diversifiées, même si certains problèmes apparaissent passablement négligés. En effet, aucune recherche inventoriée ne porte sur ces aspects: recherche appliquée et productivité, crédit, prix, fiscalité, investissement et commerce extérieur. Tous les autres secteurs spécifiques ont cependant retenu l'attention des chercheurs et ce, en nombre suffisant. Dans leur très grande majorité, ces recherches ont été initiées après 1970, par des chercheurs issus de ces institutions: l'Université Laval, l'ENAP, l'École des hautes études commerciales.

Le tableau 3 résume les caractéristiques principales des champs ainsi constitués.

\section{Les missions sociales}

Gournay établit ces sous-catégories à propos des missions sociales de l'État:

$1^{\circ} \quad$ Les actions spécifiques en matière de santé 
TABLEAU 3

Missions économiques

\begin{tabular}{|c|c|c|c|c|}
\hline & NOMBRE & CHAMPS ÉTUDIÉS & THÈMES & $\begin{array}{l}\text { INSTITUTIONS } \\
\text { DES CHERCHEURS }\end{array}$ \\
\hline $\begin{array}{l}\text { Actions spécifiques dans la } \\
\text { vie économique }\end{array}$ & 34 & $\begin{array}{l}\text { - Interventions économiques } \\
\text { de l'État }\end{array}$ & $\begin{array}{l}\text { - Ressources naturelles } \\
\text { (forêt, énergie hydro- } \\
\text { électrique) } \\
\text { - Agriculture } \\
\text { - Télécommunications }\end{array}$ & ENAP \\
\hline Autres actions spécifiques & 14 & $\begin{array}{l}\text { - Relations de travail } \\
\text { - Politiques de main- } \\
\text { d'œuvre } \\
\text { - Les partages de juridiction }\end{array}$ & $\begin{array}{l}\text { - Négociations } \\
\text { collectives } \\
\text { - Règlements de griefs } \\
\text { - Loi du salaire } \\
\text { minimum }\end{array}$ & $\begin{array}{l}\text { Université Laval (Dép. } \\
\text { des relations } \\
\text { industrielles) } \\
\text { HEC }\end{array}$ \\
\hline $\begin{array}{l}\text { La coordination générale de } \\
\text { la politique économique et } \\
\text { financière }\end{array}$ & 31 & $\begin{array}{l}\text { - L'aménagement du } \\
\text { territoire } \\
\text { - La planification } \\
\text { - La politique financière } \\
\text { - La politique économique }\end{array}$ & & $\begin{array}{l}\text { Université Laval } \\
\text { ENAP } \\
\text { HEC }\end{array}$ \\
\hline
\end{tabular}


$2^{\circ} \quad$ Les actions conduites dans le domaine du logement et de l'urbanisme

$3^{\circ}$ La défense des droits et intérêts des catégories socio-professionnelles (notamment les salariés)

$4^{\circ}$ La distribution des revenus au profit des socialement «faibles»

$5^{\circ}$ Le maintien, ou la transformation des structures sociales (politique familiale, promotion sociale, etc.).

Sous cette rubrique, quarante-six recherches ont été analysées; la plupart concernent tantôt la santé, tantôt l'habitation et le logement, tantôt les affaires sociales. Environ une recherche sur deux concerne les actions en matière de santé. En fait, vingt et une recherches ont été répertoriées et ce sont les hôpitaux, les CLSC et le ministère des Affaires sociales qui sont le plus souvent cités par les chercheurs. Ils en étudient certains aspects tandis que ces problèmes semblent être abordés d'une manière spécifique: processus décisionnel - système de gestion - pouvoir et responsabilités des employés - planification des soins de santé pour le Québec en général, ou encore pour certaines régions en particulier. Par contre, rares sont les études s'intéressant à l'évolution législative en matière de santé; de même, on se préoccupe assez peu de décrire le fonctionnement des institutions concernées. Cinquante pour cent des recherches ont été complétées après 1976 mais, quelle que soit la période d'étude, deux institutions sont particulièrement actives en matière de recherche en santé: l'ENAP et le département d'administration de la santé de l'Université de Montréal.

À propos des actions conduites dans le domaine du logement et de l'urbanisme, on a recensé huit recherches. Trois d'entre elles concernent la Société d'habitation du Québec. Quelle que soit la période d'étude (cinq recherches entre 1971 et 1975 et trois après 1975) les chercheurs, attachés à l'ENAP ou encore à l'Institut 
national de la recherche scientifique - urbanisation, s'intéressent principalement à l'analyse des politiques dans ce secteur.

Finalement quinze recherches portent sur les questions relatives à ce que Gournay appelle la distribution des revenus au profit des socialement pauvres. Les organismes fréquemment cités par les auteurs sont le ministère des Affaires sociales, les Centres de services sociaux, la Commission des affaires sociales. Ces travaux sont réalisés après 1975 et neuf d'entre eux sont complétés entre 1976 et 1980. Si au début des années soixante les chercheurs parlaient d'assistance publique, il n'en va plus de même aujourd'hui. Dorénavant, les thèmes abordés sont ceux de la réinsertion sociale et de la régulation. Les auteurs s'intéressent surtout à la gestion et à la planification des structures mises en place à la fin des années soixante. L'ENAP d'abord, l'Université Laval ensuite, demeurent les institutions les plus intéressées par ces problèmes.

Les recherches sur les missions sociales de l'État sont relativement moins importantes que celles recensées dans les champs économique et politique. On note cependant que les travaux dans le domaine de la santé sont les plus nombreux. Mais, quels que soient les thèmes, la plupart des recherches furent réalisées après 1970. Une dizaine seulement l'ont été avant cette période; elles portent essentiellement sur les problèmes de santé et de sécurité sociale, étant entendu qu'on ne s'intéresse pas encore aux questions de logement et d'habitation. Toutes périodes confondues, c'est l'ENAP et l'Université de Montréal qui ont produit le plus grand nombre de travaux relatifs aux domaines de la santé et de la sécurité sociale. Dans toutes ces études, ce sont les aspects coûts - rentabilité et gestion qui demeurent les plus fréquemment traités.

Ces divers aspects sont succinctement montrés dans le tableau 4. 
TABLEAU 4

Missions sociales

\begin{tabular}{l|c|c|c|c}
\hline & NOMBRE & CHAMPS D'ÉTUDES & THÈMES & $\begin{array}{c}\text { INSTITUTIONS } \\
\text { DES CHERCHEURS }\end{array}$ \\
\hline $\begin{array}{l}\text { Actions spécifiques en } \\
\text { matière de santé }\end{array}$ & 21 & $\begin{array}{l}\text { - Planification des soins de } \\
\text { santé }\end{array}$ & $\begin{array}{l}\text { - Les institutions de } \\
\text { santé }\end{array}$ & $\begin{array}{l}\text { ENAP, Université de } \\
\text { Montréal (dép. } \\
\text { d'administration de la } \\
\text { santé) }\end{array}$ \\
\hline $\begin{array}{l}\text { Actions conduites dans le } \\
\text { domaine du logement et de } \\
\text { l'urbanisme }\end{array}$ & 8 & - Analyse des politiques & $\begin{array}{l}\text { La Société d'habitation } \\
\text { du Québec }\end{array}$ & $\begin{array}{l}\text { ENAP } \\
\text { INRS-Urbanisation }\end{array}$ \\
$\begin{array}{l}\text { La distribution des revenus } \\
\text { au profit des socialement } \\
\text { «faibles" }\end{array}$ & 15 & $\begin{array}{l}\text { - La planification et la } \\
\text { gestion des structures } \\
\text { mises en place }\end{array}$ & $\begin{array}{l}\text { - Ministère des Affaires } \\
\text { sociales } \\
\text { - Centres des services } \\
\text { sociaux }\end{array}$ & $\begin{array}{l}\text { ENAP } \\
\text { Université Laval }\end{array}$ \\
\hline
\end{tabular}


Les missions éducatives et culturelles

Ces sous-catégories sont retenues par Gournay:

$1^{\circ} \quad$ La recherche scientifique à caractère désintéressé (recherche pure ou fondamentale).

$2^{\circ} \quad L^{\prime}$ éducation des enfants et adolescents (enseignement général, professionnel, artistique, sportif, etc.), y compris les actions en faveur de la jeunesse.

$3^{\circ} \quad$ L'organisation des loisirs et activités culturelles destinées aux adultes, y compris l'information à caractère non politique.

$4^{\circ}$ Le développement des activités artistiques: conservation du patrimoine artistique et historique; encouragement à la création des œuvres nouvelles; diffusion des œuvres d'art.

Nous y avons classé trente-trois recherches; plus de deux recherches sur trois traitent de l'éducation des enfants et adolescents. Ces travaux deviennent plus nombreux à partir de 1970, cependant que leur importance augmente entre 1976 et 1980. Les organismes étudiés sont le ministère de l'Éducation, le Conseil supérieur de l'éducation, les écoles polyvalentes. La fonction du directeur d'école reste un thème intéressant pour certains auteurs, de même que ceux des coûts de l'enseignement et des relations de travail. Ce sont les universités Laval et de Montréal qui ont initié le plus grand nombre d'études en ce domaine.

L'organisation des loisirs et des activités culturelles destinées aux adultes sont relativement peu étudiées (six recherches). Les chercheurs ont notamment regardé du côté du ministère des Affaires culturelles et de la politique culturelle québécoise. Enfin, quatre travaux portent sur la recherche scientifique, parmi lesquels deux articles ayant trait aux problèmes de la recherche relative aux administrations publiques.

En somme, les travaux relatifs à cette mission concernent surtout l'éducation des enfants et adolescents en général; de manière 
plus particulière, ils analysent ce qui relève de l'enseignement proprement dit. Mais, c'est l'éducation au niveau primaire et secondaire qui est le plus souvent prise en compte. Les autres secteurs sont peu considérés, bien que les autorités gouvernementales aient agi dans ces domaines, notamment au plan des activités culturelles et de l'organisation des loisirs.

Le tableau 5 illustre les principaux champs concernés.

À travers l'analyse des missions de l'État, on est frappé par la diversité des champs couverts par les chercheurs. Ceux-ci, en effet, sont présents dans tous les domaines d'intervention de l'État moderne. Certes, leur présence n'est pas la même partout, cependant elle demeure significative tant dans les secteurs économique et de souveraineté qu'aux plans social, éducatif et culturel.

Cette polyvalence apparaît surprenante eu égard au temps relativement court pendant lequel ce système de recherche s'est constitué. N'oublions pas qu'au début des années soixante la recherche universitaire est rare et peu développée. La réforme du système éducatif permet la mise en place d'infrastructures de recherche au sein de l'université. Un effort financier important est consenti par les différents organismes subventionnaires. La recherche en général, la recherche administrative en particulier, profitent des conditions favorables ainsi créées. L'expertise administrative se développe et couvre des secteurs extrêmement variés. D'un certain point de vue, c'est d'abord aux institutions administratives et financières auxquelles les chercheurs s'intéressent. Bon nombre d'entre eux travaillent sur le fonctionnement des institutions existantes ou à mettre à place, quelques-uns sur les modes de gestion et les systèmes de contrôle.

Mais, on réfléchit aussi sur les politiques économiques et financières de l'État. Certains secteurs de la vie économique sont étudiés plus souvent que d'autres. Les questions relatives aux ressources naturelles, à l'énergie, aux transports et aux télécommunications occupent une place relativement importante. Les 
TABLEAU 5

Les missions éducatives et culturelles

\begin{tabular}{|c|c|c|c|c|}
\hline & NOMBRE & CHAMPS ÉTUDIÉS & THÈMES & $\begin{array}{l}\text { INSTITUTIONS } \\
\text { DES CHERCHEURS }\end{array}$ \\
\hline La recherche scientifique & 4 & & & \\
\hline $\begin{array}{l}\text { L'éducation des enfants et } \\
\text { adolescents }\end{array}$ & 22 & $\begin{array}{l}\text { - La démocratisation de } \\
\text { l'enseignement } \\
\text { - Les structures } \\
\text { administratives }\end{array}$ & $\begin{array}{l}\text { — Les coûts } \\
\text { — Les écoles polyvalentes } \\
\text { — le ministère de } \\
\text { l'Éducation } \\
\text { - Le Conseil supérieur de } \\
\text { l'éducation } \\
\text { - Le directeur d'école }\end{array}$ & $\begin{array}{l}\text { Université Laval } \\
\text { Université de } \\
\text { Montréal }\end{array}$ \\
\hline $\begin{array}{l}\text { L'organisation des loisirs et } \\
\text { activités culturelles } \\
\text { destinées aux adultes }\end{array}$ & 6 & $\begin{array}{l}\text { — La politique culturelle } \\
\text { québécoise }\end{array}$ & $\begin{array}{l}\text { — Le ministère des } \\
\text { Affaires culturelles }\end{array}$ & \\
\hline
\end{tabular}


domaines de la santé et de l'éducation, quoique retenus par les chercheurs, suscitent un peu moins d'analyses. Enfin, les problèmes relatifs au logement et aux services sociaux ont, pour leur part, inspiré plusieurs travaux. Cependant, leur nombre total demeure inférieur à celui de ces derniers écrits.

La bonne santé de la recherche administrative que marque cette grande diversité des champs considérés, va de pair avec une spécialisation relative des domaines et des institutions. En effet, et les tableaux 2, 3, 4 et 5 le montrent, il y a une certaine convergence entre domaines - thèmes et, quoique dans une moindre mesure, institutions des chercheurs. Il est vrai que ces convergences sont loin d'être toujours significatives. Les caractéristiques globales qui émergent de l'analyse montrent, en effet, l'existence de champs dominants et de champs en formation. Leurs rapports aux thèmes d'étude et aux institutions ne ressortent pas toujours, ce qui est compréhensible, puisque l'analyse à laquelle nous avons procédé ne nous permettait pas d'appréhender les correspondances secondaires. En réalité, et les tableaux le montrent bien, nous nous sommes attardé aux tendances dominantes, tant au niveau des champs que des thèmes et des institutions d'appartenance des chercheurs.

On sait maintenant ce que font les chercheurs qui s'intéressent à l'administration publique, c'est-à-dire ce sur quoi ils travaillent. Extrêmement diversifiée, cette recherche porte sur ce qui existe et se transforme. Mais, elle aussi spécialisée puisque les travaux recensés montrent bien que l'on n'étudie pas tout et n'importe quoi. Qui plus est, on ne l'étudie pas n'importe comment.

\section{Les problématiques}

Pour mémoire, rappelons que nous avons retenu quatre approches susceptibles de nous guider pour la suite de l'analyse. Il est bien évident que ces dernières approches, de même que les 
distinctions que nous en faisons, sont loin d'être acceptées par tous et toutes. Néanmoins, elles demeurent des distinctions nécessaires auxquelles se réferent les spécialistes de science administrative. Nos quatre approches étudient, le processus administratif tantôt comme un fait social, tantôt comme une entité subordonnée.

\section{a) L'approche juridico-politique ${ }^{17}$}

On dit de cette approche qu'elle est institutionnelle. S'intéressant surtout aux structures, à l'action et au contrôle, elle ne permet guère que la description des phénomènes en cause. Ici, l'administration n'est pas considérée comme un acteur autonome tant il est vrai que les institutionnalistes la conçoivent comme une instance seconde, chargée de préparer et d'appliquer les décisions rendues par le pouvoir politique. Au total, cette approche donne une image tronquée des institutions et se limite à la description des phénomènes plutôt qu'à leur explication.

\section{b) L'approche économique}

Lorsque les économistes analysent l'intervention de l'État dans le processus de production ou encore de distribution, ils prennent rarement en compte le caractère particulier des institutions politico-administratives étudiées. Cependant, seuls les économistes préoccupés par la place de l'administration publique, par la spécificité de son rôle et de sa gestion ont recours à ce type d'analyse.

\section{c) L'approche managériale}

Cette approche postule qu'il existe une organisation rationnelle et donc, qu'il est possible d'atteindre un ou des objectifs selon des conditions optimales, à l'aide d'outils appropriés. Les notions

17. Ici, nous reprenons des divisions de Jacques Chevallier et Danièle Loschak, Traité de science administrative, tome 1 , op. cit. 
d'efficacité, de capacité de rendement pénètrent le secteur public. Tantôt ce dernier est analysé à travers le prisme de la satisfaction, au meilleur coût possible, des besoins de l'organisation, tantôt à travers celui de la réalisation et au moindre coût, à la fois des besoins fonctionnels de l'organisation et des objectifs sociaux fixés ${ }^{18}$. Cette «science du management» a donné lieu, déjà, à de nombreux travaux, d'abord aux États-Unis puis en Europe occidentale.

\section{d) L'approche sociologique}

La sociologie politique et la sociologie des organisations s'intéressent aux administrations publiques, mais, à partir de points de vue différents. Pour sa part, la sociologie politique, en posant l'imbrication du politique et de l'administratif reprend l'idée selon laquelle le politique et l'administratif constituent les deux faces cachées d'une seule et même chose. Ainsi, la sociologie politique s'intéresse au système politico-administratif dans l'ensemble de son fonctionnement.

Quant à la sociologie des organisations, elle prend en compte les motivations, les comportements, de même que les stratégies des divers acteurs. De plus, elle regarde l'environnement externe comme pouvant exercer une détermination relative sur la configuration des organisations. Certes, ces quatre approches n'épuisent pas la totalité des courants théoriques auxquels les chercheurs ont recours lorsqu'ils étudient les administrations publiques. Elles constituent néanmoins les approches dominantes du champ, par ailleurs fécond lorsque sont considérés les échanges de la linguistique, de la psychologie, de l'histoire, de la philosophie et ainsi de suite.

Les chercheurs, dont l'axe dominant sont les institutions administratives et financières, privilégient deux approches: l'ap-

18. Sur cette question, voir Michel Bellavance, «Valeurs et politiques: considérations théoriques et problématiques pratiques", Revue canadienne de science politique, XVI:3, septembre 1983, pp. 519-533. 
proche juridico-politique d'abord, l'approche managériale ensuite. Si l'approche juridico-politique domine, on observe un recours grandissant aux approches managériale et sociologique, principalement à partir de 1970. Si les chercheurs utilisant l'approche sociologique étudient notamment les questions liées à la consultation et à la participation, on retient l'approche managériale davantage pour l'analyse des problèmes liés au contrôle. Ces convergences dans le champ, dominant parce qu'on y a classé près d'une recherche recensée sur deux, méritent un peu plus d'attention. En effet, des différences quant à la manière de poser les questions et de les traiter ressortent, et un nouveau discours prend forme. Il faut dire que l'approche juridico-politique, fréquemment retenue, traite les problèmes administratifs en utilisant ces notions: évolution, participation, personnel public, pour ne mentionner que ceuxlà. Par contre, la prise en compte des questions relatives à la prise de décision, à l'efficacité, à la rationalité, à l'imputabilité, aux programmes, etc., est peu fréquente avant le début des années soixante-dix. Ce nouveau discours émerge surtout des recherches utilisant ces problématiques: sociologique et managériale. Le recours plus fréquent à ces dernières problématiques modifie quelque peu le corpus de la recherche. Car, si les chercheurs continuent de se pencher sur l'aspect institutionnel, ils ne s'y limitent plus. Ils étudient l'administration publique à travers des processus et des phénomènes, laissant supposer que son contenu réel s'est modifié. Ce faisant, on reconnaît que l'administration est bien autre chose qu'une organisation rationnelle de services et de fonctions et que son fonctionnement n'est pas aussi formaliste que certaines théories juridiques l'ont d'abord laissé supposer.

Les travaux relatifs aux interventions de l'État dans la production des biens ou encore dans leur répartition se limitent, eux aussi, à deux courants théoriques: les approches managériale et économique. Mais, la nature spécifique des institutions en cause n'induit guère de différence dans la façon dont les chercheurs 
abordent ces questions. En effet, il est assez rare que le caractère politique de décisions ayant de fortes incidences économiques soit traité comme tel. Jusqu'à maintenant en tout cas, peu de travaux de ce type ont considéré le caractère particulier de l'État. Ses prérogatives de puissance publique en font un acteur influent dans la recherche et la mise en œuvre d'outils économiques de gestion et de planification.

Dans le domaine social par contre, certains travaux sociologiques émergent. Les questions auxquelles s'intéressent les auteurs portent surtout sur la participation et le processus décisionnel. Cela s'explique sans doute par la mise en place, dans le domaine de la santé et du logement, de structures nouvelles favorisant la participation des usagers.

Enfin, dans le domaine de l'éducation, on a plus souvent recours aux approches juridico-politique et managériale qu'à la sociologie des organisations. Les auteurs examinent donc les problèmes relatifs à ce secteur, tant à partir de l'institution ellemême qu'à travers un questionnement de type rationaliste et techniciste.

Le corpus de connaissance qui se dégage ici montre bien l'existence de secteurs dominants, plus ou moins développés, auxquels correspondent des problématiques ou des champs théoriques. Ces derniers champs théoriques reposent sur des prémisses différentes, d'où une conception différenciée des chercheurs, relative à l'étude des phénomènes administratifs.

La conception la plus ancienne et la plus courante est celle d'une administration publique différente du politique autant que des administrations privées. L'objet d'étude est l'administration tandis que son fonctionnement est abordé à travers ce qui est donné par les textes. Tout autre est la science du management selon laquelle il y a identité entre le secteur public et le secteur privé. On pose la possibilité de transposer ce qui se passe dans le privé, au secteur public, de manière à améliorer le fonctionnement 
du système. Cette approche est plus normative que la précédente et repose tant sur les méthodes scientifiques d'aide à la décision que sur les méthodes d'analyse d'une fonction précise. Enfin, la sociologie reconnaît que la spécificité de l'administration lui vient du politique, d'où la difficulté d'isoler, dans un à-part, les phénomènes administratifs.

Certes, ces conceptions n'épuisent pas la totalité du regard des chercheurs sur l'administration publique, cependant elles rendent compte des problématiques dominantes. Ces dernières caractérisent le corpus de connaissance qui s'est développé depuis 1960, affirmant ainsi la présence de pratiques différenciées dans la recherche administrative.

\section{Les enjeux de la recherche}

L'analyse précédente a révélé que la recherche en administration publique est diversifiée, spécialisée et différenciée. On sait maintenant que la connaissance qu'ont les chercheurs de l'administration publique n'est pas du tout celle qui prévalait au début des années soixante. Il est certain qu'au sein de l'administration elle-même, de nombreux changements sont intervenus. Il est vrai également que le développement de la recherche en sciences sociales a largement profité aux pouvoirs publics en devenant, dans plusieurs cas, une source d'expertise non négligeable.

Tous ces changements ne nous conduisent-ils pas à chercher l'existence de liens entre la réforme administrative et la recherche elle-même? Peut-on croire, en effet, que l'adaptation du secteur public aux nouvelles réalités fut possible sans qu'un effet de retour s'exerce sur la connaissance qui en était donnée? À son tour, cette connaissance n'a-t-elle pas constituée une nouvelle réalité administrative, limitée aux contours visibles de l'administration?

S'il est difficile de répondre directement à ces questions il est néanmoins possible de réfléchir davantage sur le contenu de 
la recheche complétant ainsi l'analyse précédente. Demandonsnous donc si le discours des chercheurs présente une spécificité vis-à-vis du fonctionnement réel des administrations ou encore si ce discours établit des liens étroits avec les différentes facettes de la réalité administrative. La relation entre institutions de recherche et approches des chercheurs prend ici valeur d'indice, d'autant qu'il semble exister un certain rapport entre l'institution d'appartenance du chercheur et le courant théorique auquel il se réfere.

La réflexion précédente a montré que les universités n'ont pas le monopole de la recherche sur l'administration publique québécoise, bien que les universités Laval et de Montréal occupent un secteur relativement important du champ. En effet, la création de l'ENAP, combinée à la mise sur pied de centres de recherche, a modifié le «savoir» et le «faire»sur l'administration.

L'on sait que c'est au cours des années soixante et même si les recherches sont quantitativement plus limitées qu'aujourd'hui, que les infrastructures de recherche se développent. Ces dernières infrastructures gravitent autour des universités de Montréal et de Laval, tandis que la région de Montréal est légèrement prédominante au cours de ces années. Les chercheurs ont majoritairement recours à ces deux approches: d'abord l'approche juridico-politique, ensuite l'approche managériale. Des liens entre les thèmes étudiés et les approches retenues par les chercheurs ressortent également. Tandis que l'approche juridico-politique traite des questions concernant les institutions, leur fonctionnement, les problèmes de travail et d'administration, l'approche managériale par contre, analyse les politiques gouvernementales ainsi que les problèmes administratifs qui en découlent. En réalit, près de $80 \%$ des recherches analysées au cours de ces années utilisent un cadre d'analyse tantôt juridicopolitique, tantôt managérial. Tandis que les départements de science politique de Laval et de Montréal se penchent surtout sur les problèmes institutionnels, les centres de recherche, s'intéressent, quant à eux, aux politiques gouvernementales et à leurs effets. 
Certes, au cours des années soixante, l'approche juridicopolitique domine, puisque les chercheurs l'utilisent une fois sur deux. Mais, il n'en va plus de même après le début des années soixante-dix, alors que près de $50 \%$ des travaux sont réalisés à l'aide d'une approche managériale. Ces derniers travaux concernent principalement les techniques administratives et les politiques gouvernementales. Bien sûr, on continue d'étudier les institutions politico-administratives et les problèmes d'organisation et d'administration qui en découlent. Mais, ces préoccupations sont relativement moins importantes que lors de la période précédente, puisque seulement $25 \%$ des études concernent ce secteur. Il semble bien que les domaines des chercheurs changent tandis que la région de Québec apparaît devoir concentrer la plupart des recherches relatives aux problèmes administratifs. Même la création et le développement de l'Université du Québec à Montréal ne parviennent guère à modifier cette situation. En outre, les centres de recherche spécialisés ainsi que l'École nationale d'administration publique occupent désormais un espace de plus en plus important du champ.

C'est ainsi qu'une partie de la recherche sur les phénomènes administratifs se déplace graduellement du secteur universitaire vers les centres de recherche spécialisés. Ces derniers centres s'intéressent à des domaines précis et développent une grande expertise en la matière, notamment l'Institut national de la recherche scientifique - urbanisation, le Centre de recherche en développement économique. En outre, tous ces changements s'accompagnent d'un intérêt croissant pour les problèmes économiques que les chercheurs analysent à travers une problématique économique classique dès lors que la spécificité des institutions concernées n'est guère prise en compte.

En un sens, on peut croire qu'il s'agit là d'un système de recherche sensiblement différent de celui qui existait au cours des années soixante. À cette époque en effet, les universités occupent 
la quasi-totalité du champ, tandis que le discours dominant demeure celui d'une administration distincte, spécifique et subordonnée. Si par la suite les chercheurs se mettent à réfléchir sur la portée des changements intervenus, leurs préoccupations théoriques s'inscrivent dans un ensemble cohérent et rendent compte des transformations administratives. Ces dernières transformations, censées moderniser l'institution administrative, renforcent la spécialisation. Parallèlement, le discours de l'État révèle une étroite imbrication avec celui des chercheurs que traduit la référence sans cesse renouvelée à la rationalité, à l'efficacité. En réalité, le nouveau discours de l'État reflète une relation constante à la norme; d'abord juridique, cette dernière glisse de plus en plus vers un contenu de type technicien, marquant ainsi la transformation dans le fonctionnement et les finalités de l'appareil administratif.

Le renforcement de la spécialisation, combiné à une rationalité d'efficacité, assure le repérage et le quadrillage des phénomènes administratifs que l'on découpe en domaines précis et spécialisés. L'appareil administratif ainsi modernisé favorise la mise en place et le développement d'infrastructures de recherche spécialisées. Ces dernières infrastructures opèrent la mise en force d'un discours scientifique qui semble emprunter des voies multiples: planification, participation, décentralisation, consultation, rationalisation budgétaire, contrôle, décision, technologie, efficacité, etc. D'un côté il y a une administration transparente et démocratique, de l'autre se dessine une administration secrète, fermée et rationnelle. Mais, ces contradictions dans' le discours ne sont qu'apparentes. En effet, n'est-ce pas une des caractéristiques des administrations modernes que de s'ouvrir aux administrés tout en accroissant ses pouvoirs, étant donné son importance dans le contrôle des nouvelles ressources d'expertise?

Le système de recherche découlant de l'étude des phénomènes administratifs se caractérise donc par l'existence de centres de recherche spécialisés dans et hors de l'université. Le discours des 
chercheurs, complexe et multiple, laisse supposer que l'administration moderne relève de rationalités plurielles. Ce discours traduit un processus de régulation compliqué, exprimant par là une transformation de la logique sociale. Cette dernière marque l'action des administrations modernes qui, à leur tour, favorisent sa reproduction.

On le voit, et l'évolution du système de recherche que nous venons d'analyser le montre, il devient difficile de prétendre que l'administration se situe dans un rapport d'extériorité avec le socio-politique. En effet, l'administration québécoise, que traduit la spécificité de notre système de connaissance, présente des liens étroits avec les transformations profondes ayant marqué l'État et la société. En un sens, il est possible de retracer ces changements à travers l'analyse de la recherche administrative au cours des vingt dernières années (1960-1980). Mais, et ceci peut paraître contredire nos propos précédents, la recherche administrative n'at-elle pas eu tendance à couper la réalité administrative de la réalité politique? En d'autres termes, est-ce que cette recherche n'est pas finalement partielle dès lors qu'elle prend rarement en compte le statut d'appareil d'État de l'administration? En fin de compte, et ceci n'a rien d'exceptionnel, la recherche administrative s'intéresse aux institutions plutôt qu'aux phénomènes, aux méthodes de gestion plutôt qu'à la compréhension de leur développement et de leur mise en application. Ce faisant, elle permet aux autorités politiques un regard sur leur action et, du même coup, devient un enjeu politique. 